\title{
Carcinoids and Capsules: A Case Series Highlighting the Utility of Capsule Endoscopy in Patients With Small Bowel Carcinoids
}

\author{
Michael G. Noujaim a, e, f, Jonathan Green ${ }^{\mathrm{b}, \mathrm{e}}$, May Min ${ }^{\mathrm{c}}$, Christopher R. Schlieve ${ }^{\mathrm{b}}$, \\ Krunal Patel ${ }^{\mathrm{c}, \mathrm{d}}$, Mitchell Cahan ${ }^{\mathrm{b}}$, David Cave, d
}

\begin{abstract}
Background: Neuroendocine tumors (NETs) or carcinoids arise at many different sites of the gastrointestinal tract. The small intestine is the most common site for NETs. Diagnosing small bowel carcinoids remains challenging given their non-specific presentations and the overall low incidence of small bowel tumors. Video capsule endoscopy (VCE) has significanly improved our ability to detect small bowel malignancies. We explore the value of VCE in the initial workup and management of a series of small bowel carcinoid patients.
\end{abstract}

Methods: We retrospectively analyzed adult patients undergoing surgical management for small bowel lesions from July 2005 to September 2015 at a tertiary care center. Patient characteristics, presenting symptomatology, diagnostic workup and surgical management were analyzed among patients with histologically confirmed small bowel carcinoid tumors.

Results: Our study identified 16 patients treated surgically for small bowel carcinoids. The majority of patients $(87.5 \%)$ presented with either occult gastrointestinal bleeding or anemia. Most patients $(87.5 \%)$ were initially evaluated with various endoscopic and imaging modalities before all ultimately undergoing surgery. Seventy-five percent of patients had a VCE, with $83.3 \%(10 / 12)$ having positive findings that correlated with intraoperative findings compared to $62.5 \%(5 / 8)$ with computed tomography scan, $21.4 \%$ (3/14) with colonoscopy, $44 \%$ (4/9) with deep enteroscopy, and $0 \%(0 / 9)$ with esophagogastroduodenoscopy (EGD).

Manuscript submitted November 20, 2017, accepted December 12, 2017

aDepartment of Internal Medicine, Duke University Medical Center, Durham, NC, USA

bepartment of Surgery, University of Massachusetts Medical School, Worcester, MA, USA

'Department of Internal Medicine, University of Massachusetts Medical School, Worcester, MA, USA

${ }^{\mathrm{d} D i v i s i o n}$ of Gastroenterology, University of Massachusetts Medical School, Worcester, MA, USA

${ }^{\mathrm{e}}$ These authors contributed equally to this work.

${ }^{f}$ Corresponding Author: Michael G. Noujaim, Department of Internal Medicine, Duke University Medical Center, Durham, NC, USA.

Email:mgn9@duke.edu

doi: https://doi.org/10.14740/gr937w
Conclusions: In the absence of any contraindications, VCE is an effective endoscopic modality in the diagnostic workup of small bowel NETs. Furthermore, positive VCE findings appear to highly correlate with surgical findings, thus suggesting a valuable role for VCE in the initial surgical assessment of patients with small bowel NETs.

Keywords: Carcinoid; Neuroendocrine tumors; Small bowel; Video capsule endoscopy; Surgical resection

\section{Introduction}

Neuroendocrine tumors (NETs) - formerly known as carcinoids - arise at many sites throughout the body, including, the lungs, gastrointestinal (GI) tract, hepatobiliary system, pancreas, and reproductive organs [1]. The GI tract is the most common site for NETs, accounting for $60 \%$ of tumors, with the small bowel comprising $42 \%$ of all GI NETs $[1,2]$. Diagnosing small bowel NETs remains challenging given their non-specific presentations and low incidence, accounting for approximately $1.4 \%$ of all GI malignancies [3]. Despite their low incidence, neoplasms of the small intestine are responsible for $75 \%$ of the symptomatic small bowel lesions requiring surgical intervention $[4,5]$, making their timely detection and accurate localization highly pertinent.

Computed tomography (CT) and magnetic resonance imaging (MRI) are the most commonly used modalities for the initial evaluation of suspected small bowel NETs given their good sensitivity, specificity, and availability $[2,6]$. Nevertheless, despite their overall relatively high sensitivities, both CT and MRI have been limited in their usefulness for detecting GI NETs, particularly in the small intestine [2]. In recent years, video capsule endoscopy (VCE) has significanly improved the ability to detect small bowel malignancies with multiple studies demonstrating a clear superiority for VCE in detecting small bowel tumors compared to radiologic imaging [7]. VCE is most commonly indicated in cases of overt or occult obscure GI bleeding (OGIB), which is a common presenting symptom of small bowel malignancies, including NETs [7]. Current literature demonstrates that VCE is more accurate in detecting malignant and benign small bowel masses compared to $\mathrm{CT}$ and barium studies [8]. However, current literature does 
Table 1. Patient Characteristics

\begin{tabular}{ll}
\hline & All patients $(\mathbf{n}=\mathbf{1 6})$ \\
\hline Age (mean, years) & $64 \pm 12.5$ \\
Male (\%) & $7(44)$ \\
Race (\%) & $16(100)$ \\
$\quad$ Caucasian & \\
Co-morbidities & $28.2 \pm 10.3$ \\
$\quad$ Body mass index $\left(\mathrm{kg} / \mathrm{m}^{2}\right)$ & $6(37.5)$ \\
Tobacco use & $3(19)$ \\
History of cancer & $1(6.25)$ \\
Presentation & $14(87.5)$ \\
Acute & $1(6.25)$ \\
Chronic & \\
Acute-on-chronic & $9(56.2)$ \\
Symptoms & $5(31.2)$ \\
GI bleed & $4(25)$ \\
Anemia & $3(19)$ \\
Nausea/vomit & $6(37.5)$ \\
Diarrhea & \\
Abdominal pain & \\
\hline
\end{tabular}

Values are $\mathrm{N}(\%)$ or mean (standard deviation) unless otherwise specified.

not specifically demonstrate the utility of VCE in identifying small bowel carcinoids.

In this study, we retrospectively explore the value and utility of VCE in the initial workup and management of 16 surgically confirmed small bowel NET cases.

\section{Patients and Methods}

\section{Patient population}

A retrospective chart analysis was performed on adult patients ( $\geq 18$ years of age) who underwent open or laparoscopic abdominal surgery for suspected small bowel lesions in the jejunum or ileum at a tertiary care center from July 2005 to September 2015. Only surgically resected cases with histologically proven NETs were included in the study. Study design and conduct was reviewed and approved by the University of Massachusetts Medical School's institutional review board.

\section{Data collection}

Medical charts were reviewed for patient demographic characteristics, co-morbidities, pre-operative imaging/workup, endoscopic interventions, hospital course and disposition. Study data were collected in REDCap (Research Electronic Data Capture) electronic data capture tools hosted at the University
Table 2. Endoscopy and Imaging Findings

\begin{tabular}{|c|c|c|}
\hline & \multicolumn{2}{|c|}{ All patients $(n=16)$} \\
\hline & Overall & Positive finding $\S$ \\
\hline \multicolumn{3}{|l|}{ Endoscopy } \\
\hline EGD & $9(56)$ & $0(0)$ \\
\hline Colonoscopy & $14(87)$ & $3(21.4)$ \\
\hline Video capsule endoscopy & $12(75)$ & $10(83.3)$ \\
\hline Deep enteroscopy & $9(56)$ & $4(44)$ \\
\hline None & $2(12.5)$ & - \\
\hline \multicolumn{3}{|l|}{ Radiology } \\
\hline X-ray & $2(12.5)$ & $0(0)$ \\
\hline $\mathrm{CT}$ & $8(50)$ & $5(62.5)$ \\
\hline CTE & $4(25)$ & $3(75)$ \\
\hline MRE & $2(12.5)$ & $1(50)$ \\
\hline None & $4(25)$ & - \\
\hline
\end{tabular}

Values are $\mathrm{N}(\%)$ unless otherwise specified. §Positive finding that correlated to operative findings/location.

of Massachusetts Medical School. REDCap is a secure, webbased application designed to support data capture for research studies, providing 1) an intuitive interface for validated data entry; 2) audit trails for tracking data manipulation and export procedures; 3) automated export procedures for seamless data downloads to common statistical packages; and 4) procedures for importing data from external sources [9].

\section{Results}

\section{Patient characteristics}

Our study identified 16 patients treated surgically for small bowel NETs. The mean age of the study sample was approximately 64 years old, over half were female $(56 \%)$, and all were Caucasian. The majority of patients $(87.5 \%)$ presented with chronic symptoms and the most common presenting symptoms were OGIB and anemia (87.5\%). Over one-third $(37.5 \%)$ of patients had abdominal pain associated with obstructive symptoms (diarrhea, nausea, and vomiting) on presentation (Table 1).

\section{Endoscopy and imaging findings}

Almost all of the patients $(87.5 \%)$ were initially evaluated with one or more of the endoscopic and/or imaging modalities listed in Table 2. Notably, three-quarters (75\%) were evaluated using VCE. All 16 patients ultimately underwent surgical therapy. When retrospectively compared to the other endoscopic and imaging modalities, VCE not only detected small bowl tumors more often than the other studies, it also was the only modality whose positive findings most highly correlated with intraoperative findings in terms of location, tumor morphology, and 

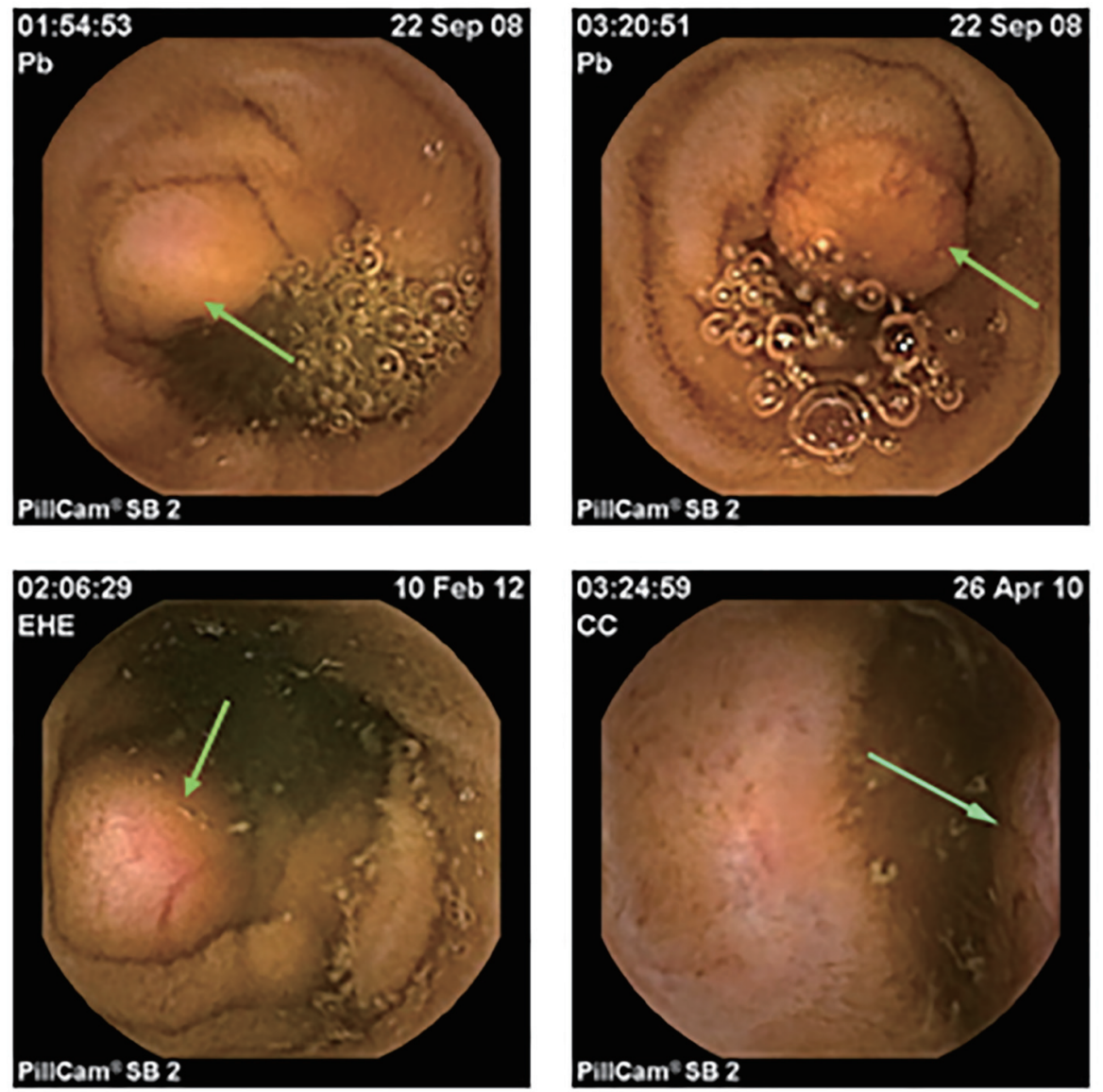

Figure 1. Examples of small bowel neuroendocrine tumors (carcinoids) detected on video capsule endoscopy.

number of lesions (Fig. 1).

\section{Operative details and final histopathology}

Over two-thirds (68.7\%) of patients underwent a small bowel resection with primary anastomosis, four $(25 \%)$ had an ileocecectomy due to the ileocecal location of their tumor(s) and one patient had a small bowel resection and a cecectomy due to a multifocal lesion. Only five patients had a pre-operative clinical diagnosis of NET; nevertheless, all 16 patients had a final postoperative, histologically proven diagnosis of GI NET (Table 3).

Upon surgical resection, $75 \%$ of patients had multiple lesions. Two patients had only a single jejunal mass, 11 patients had only ileal involvement and three patients had both jejunal and ileal lesions. Final histopathologic examination revealed that all specimens invaded at least the submucosa and $75 \%$ had either vascular, lympatic or neural invasion.

\section{Follow-up and survival}

There was only one 30-day readmission for wound abscess and Clostridium difficile infection. Median follow-up time was 53.5 months $(6-98)$ with over two-thirds $(68.7 \%)$ of patients being asymptomatic at the time of follow-up. Four patients $(25 \%)$ had diarrhea and three patients $(18.75 \%)$ had either obstruction, nausea/vomitting or abdominal pain at

Table 3. NET Characteristics in the Surgical Pathology Report

\begin{tabular}{|ll}
\hline & All patients $(\mathbf{n}=\mathbf{1 6})$ \\
\hline $\begin{array}{l}\text { Depth of invasion } \\
\text { Serosa }\end{array}$ & $6(37.5)$ \\
Muscularis & $7(43.7)$ \\
Submucosa & $3(18.7)$ \\
Mucosa & $0(0)$ \\
Neurovascular invasion & \\
Vascular & $6(37.5)$ \\
Local lymph node(s) & $11(68.7)$ \\
Neural & $1(6.25)$ \\
No invasion & $4(25)$ \\
Final histologic diagnosis & \\
Carcinoid (NET) & $16(100)$ \\
\hline
\end{tabular}

Values are $\mathrm{N}(\%)$ unless otherwise specified. 
Table 4. Follow-Up and Survival

\begin{tabular}{ll}
\hline & All patients $(\mathbf{n}=\mathbf{1 6})$ \\
\hline 30-day readmission & $1(6.25)$ \\
30-day mortality & $0(0)$ \\
Reoperation & $3(18.7)$ \\
Median follow-up in months (range) & $53.5(6-98)$ \\
Symptoms at follow-up & \\
$\quad$ Obstructive & $1(6.25)$ \\
$\quad$ Diarrhea & $4(25)$ \\
$\quad$ Nausea/vomit & $1(6.25)$ \\
$\quad$ Abdominal pain & $1(6.25)$ \\
$\quad$ None & $11(68.7)$ \\
Survival at time of chart review & $14(87.5)$ \\
Cause of death & \\
$\quad$ Metastatic disease & $2(12.5)$ \\
\hline
\end{tabular}

Values are $\mathrm{N}(\%)$ or mean (standard deviation) unless otherwise specified.

follow-up. All 16 patients were alive at 30-day follow-up. At the time of our chart review, only two patients had died due to metastatic disease - notably, both patients initially presented with obstructive symptoms, received no endoscopic interventions, and were found to have mutliple lesions upon surgical resection. Nevertheless, both patients were alive, although symptomatic (diarrhea/vomiting) at $>80$ months follow-up. In general, even though small and metastatic to local nodes, none of the NETs in this cohort had recurred after surgical resection (Table 4).

\section{Discussion}

In this retrospective case series, we demonstrate the usefulness of VCE in the initial workup and detection of small bowel NETs. Over the past decade, VCE has surfaced as a valuable tool in the diagnosis of small bowel lesions, particularly in patients presenting with OGIB and/or with clinical suspicion for small bowel malignancies $[8,10,11]$. By enabling visualization of the full length of the small intestines, VCE allows for a diagnosis and focused treatment. In a recent prospective study comparing wireless capsule endoscopy with intraoperative enteroscopy in patients with OGIB, one study found VCE had a sensitivity, specificity, and positive and negative predictive value of capsule endoscopy were $95 \%, 75 \%, 95 \%$, and $86 \%$, respectively [12]. Furthermore, a prospective study involving 28 patients admitted with OGIB compared VCE, CT angiography and standard angiography in detecting a bleeding source. The study found that VCE detected a source of bleeding in $72 \%$ of patients, compared to $24 \%$ by CT angiography and $56 \%$ by standard angiography [13]. In addition, VCE has been shown to have higher diagnostic yields compared to current imaging and endoscopic modalities $[8,10,11]$. For instance, a recent meta-analysis by Triester et al looking at three studies with a total of 88 patients revealed a yield of $67 \%$ for VCE compared to $8 \%$ for small bowel radiographic studies [11]. The same analysis found VCE to be superior to both push enteroscopy and small bowel barium imaging for the diagnosis of clinical pathology in patients with OGIB [11]. Another recent retrospective cohort demonstrated that benign and malignant small bowel masses were more frequently identified on VCE compared to barium studies and CT findings [8].

In our present study, $75 \%(12 / 16)$ of patients had a VCE, with $83.3 \%(10 / 12)$ having positive findings that correlated with intraoperative findings compared to $62.5 \%$ (5/8) with CT scan, 21.4\% (3/14) with colonoscopy, 44\% (4/9) with deep enteroscopy, and $0 \%(0 / 9)$ with esophagogastroduodenoscopy (EGD). This is consistent with current literature that demonstrates high diagnositic yield of $\operatorname{VCE}[8,10,11]$. As a result, our findings are in line with the growing body of literature highlighting the advantage and utility of VCE compared to other endoscopic and imaging modalites in detecting and diagnosing small bowel pathology. Furthermore, the natural history of small bowel NETs even with local metastases appears different from those presenting with pain, diarrhea and obstruction. Our findings suggest that small bowel NETs may behave differently from NETs arising in other anatomic sites.

\section{Conclusion}

In patients for whom there are no contraindications, VCE is an effective, non-invasive, and reliable endoscopic modality in the diagnostic workup of small bowel tumors, including NETs. Furthermore, positive VCE findings appear to highly correlate with surgical findings, thus suggesting a valuable role for VCE in the initial surgical assessment of patients with small bowel NETs.

\section{References}

1. Godwin JD, 2nd. Carcinoid tumors. An analysis of 2,837 cases. Cancer. 1975;36(2):560-569.

2. ElGuindy YM, Javadi S, Menias CO, Jensen CT, Elsamaloty H, Elsayes KM. Imaging of secretory tumors of the gastrointestinal tract. Abdom Radiol (NY). 2017;42(4):1113-1131.

3. Herlinger H, Maglinte DDT, Birnbaum BA, eds. Clinical imaging of the small intestine. 2nd ed. New York, NY: Springer-Verlag, 2001; 575.

4. Herbsman H, Wetstein L, Rosen Y, Orces H, Alfonso AE, Iyer SK, Gardner B. Tumors of the small intestine. Curr Probl Surg. 1980;17(3):121-182.

5. Barclay TH, Schapira DV. Malignant tumors of the small intestine. Cancer. 1983;51(5):878-881.

6. Cloyd JM, Poultsides GA. Non-functional neuroendocrine tumors of the pancreas: Advances in diagnosis and management. World J Gastroenterol. 2015;21(32):95129525.

7. Hara AK, Leighton JA, Sharma VK, Heigh RI, Fleischer DE. Imaging of small bowel disease: comparison of capsule endoscopy, standard endoscopy, barium examina- 
tion, and CT. Radiographics. 2005;25(3):697-711; discussion 711-698.

8. Hara AK, Leighton JA, Sharma VK, Fleischer DE. Small bowel: preliminary comparison of capsule endoscopy with barium study and CT. Radiology. 2004;230(1):260265.

9. Harris PA, Taylor R, Thielke R, Payne J, Gonzalez N, Conde JG. Research electronic data capture (REDCap)--a metadata-driven methodology and workflow process for providing translational research informatics support. J Biomed Inform. 2009;42(2):377-381.

10. Mishkin DS, Chuttani R, Croffie J, Disario J, Liu J, Shah R, Somogyi L, et al. ASGE Technology Status Evaluation Report: wireless capsule endoscopy. Gastrointest Endosc. 2006;63(4):539-545.
11. Triester SL, Leighton JA, Leontiadis GI, Fleischer DE, Hara AK, Heigh RI, Shiff AD, et al. A meta-analysis of the yield of capsule endoscopy compared to other diagnostic modalities in patients with obscure gastrointestinal bleeding. Am J Gastroenterol. 2005;100(11):2407-2418.

12. Hartmann D, Schmidt H, Bolz G, Schilling D, Kinzel F, Eickhoff A, Huschner W, et al. A prospective two-center study comparing wireless capsule endoscopy with intraoperative enteroscopy in patients with obscure GI bleeding. Gastrointest Endosc. 2005;61(7):826-832.

13. Saperas E, Dot J, Videla S, Alvarez-Castells A, Perez-Lafuente M, Armengol JR, Malagelada JR. Capsule endoscopy versus computed tomographic or standard angiography for the diagnosis of obscure gastrointestinal bleeding. Am J Gastroenterol. 2007;102(4):731-737. 